\title{
PhD theses in constraints 2012-2015
}

\author{
Guido Tack $^{1} \cdot$ Christopher Mears ${ }^{1}$
}

Published online: 10 September 2015

(C) Springer Science+Business Media New York 2015

A significant part of the research in any scientific community is carried out by $\mathrm{PhD}$ students. Due to the nature of a $\mathrm{PhD}$ project, these students often drive innovation and are at the forefront of research in their field. Some of their work is of course published in conference papers and journal articles, but a thesis offers the opportunity to work on a topic in much more depth and breadth than what is typically considered "publishable".

The goal of this new initiative is to highlight recent work by $\mathrm{PhD}$ students in the area of Constraints. We believe that their work deserves a prominent and easily accessible place in our community. The Association for Constraint Programming has therefore launched a $\mathrm{PhD}$ Thesis Archive (http://www.a4cp.org/theses) to collect and promote theses in our field of reserach. The Constraints journal has kindly agreed to publish a collection of abstracts, and as a service to the community, make them available free of charge.

For this first edition, we collected abstracts of $\mathrm{PhD}$ theses from students who graduated in 2012-2015. The topics span the full spectrum of research in our diverse field, from theoretical to applied, from soft constraints to hard, and with contributions branching out into neighbouring fields. The collection includes research that has received several best paper and best thesis awards, and has authors from every continent (except Antarctica). This should only be the first in a series, so make sure you submit your own abstract, and encourage your colleagues and students to do the same!

Guido Tack and Christopher Mears (guest editors)

Guido Tack

guido.tack@monash.edu

Christopher Mears

chris.mears@monash.edu

1 Monash University, Caulfield East, VIC 3145, Australia 